\title{
A phenotypic screening platform to identify small molecule modulators of Chlamydomonas reinhardtii growth, motility and photosynthesis
}

Simon E Alfred ${ }^{1,2,3}$, Anuradha Surendra ${ }^{1,2,4}$, Chris Le', Ken Lin' ${ }^{1}$, Alexander Mok', lain M Wallace ${ }^{1,6}$, Michael Proctor ${ }^{5}$, Malene L Urbanus ${ }^{1,4}$, Guri Giaever ${ }^{1,3,4}$ and Corey Nislow ${ }^{1,2,3^{*}}$

\begin{abstract}
Chemical biology, the interfacial discipline of using small molecules as probes to investigate biology, is a powerful approach of developing specific, rapidly acting tools that can be applied across organisms. The single-celled alga Chlamydomonas reinhardtii is an excellent model system because of its photosynthetic ability, cilia-related motility and simple genetics. We report the results of an automated fitness screen of 5,445 small molecules and subsequent assays on motility/phototaxis and photosynthesis. Cheminformatic analysis revealed active core structures and was used to construct a naïve Bayes model that successfully predicts algal bioactive compounds.
\end{abstract}

\section{Background}

Chemical biology uses small molecules to study and manipulate biological systems (reviewed in [1]). By altering an organisms' normal state, and thereby affecting growth or development, we can learn about the contributions of the perturbed processes to the organisms' fitness, physiology and homeostasis. The approach is analogous to genetic manipulation to produce an observable phenotype (reviewed in [2]). Small molecules, in addition to complementing genetic perturbations, have several advantages: they can be applied at varied concentrations, during different stages of development and on specific tissues, to different organisms, and their (frequently) rapid reversibility can be used to modulate dynamic processes.

The single-celled green alga Chlamydomonas reinhard$t i i$, often referred to as the 'green yeast' [3], is a powerful model organism that can be easily manipulated, with straightforward genetics and a wide range of informative phenotypes. Furthermore, its biology is relevant to both plants and animals and to human disease, for example, ciliopathies [4,5]. Key discoveries have been made from study of Chlamydomonas' chloroplast [6,7] as well as

\footnotetext{
* Correspondence: corey.nislow@utoronto.ca

'Donnelly Centre for Cellular and Biomolecular Research, University of

Toronto, 160 College Street, Toronto, Ontario M5S 3E1, Canada

Full list of author information is available at the end of the article
}

components of its light perception [8] and light response complexes [9]. Evolutionary studies have focused on evolution of fitness under selective pressures (elevated $\left[\mathrm{CO}_{2}\right]$, sexual/asexual populations) and the evolution of multicellularity [10-13]. Chlamydomonas has provided a wealth of data on flagellar formation and function and intraflagellar transport was first observed in Chlamydomonas [14]. As one of the few model organisms with motile flagella, combined with renewed interest in primary cilia and flagellar/ ciliary disorders [15], Chlamydomonas is a clinically important test system for perturbation.

Chemical perturbation studies on green algae have focused primarily on inhibitors of photosynthesis, particularly for agricultural and research applications $[16,17]$. Chlamydomonas' chemical sensitivity has also been exploited to assess environmental toxins such as cadmium and the herbicide fluoxypyr $[18,19]$, providing information on small molecule bioaccumulation and uptake [20,21]. In addition, chemical genetic screens have tested small molecules for their ability to alter motility $[22,23]$, phototaxis [24], and flagellar formation and regeneration [25,26]. However, a large-scale, comprehensive screen for small molecule inhibitors of fitness has, to our knowledge, not yet been reported. Our experience with yeast chemical perturbation has shown that data derived from simple fitness screens are quite valuable, with growth being the ultimate 'integrative phenotype'. Information at this stage can 
be invaluable for facilitating genetic and genomic approaches to determine possible mechanisms of action of small molecule growth inhibitors $[27,28]$.

Here we developed a small molecule screen at very highthroughput to identify fitness inhibitors of $C$. reinhardtii, based on two screens, one for long-term fitness in the presence of compound, and another for phenotypic effects on photosynthesis and motility/phototaxis using short-term exposure. We demonstrate the effectiveness of Chlamydomonas as a chemical biology subject, and define and model physiochemical parameters that characterize small molecule activity on Chlamydomonas. As part of this study we generated a chemical biology Chlamydomonas resource searchable by identifier or structure and that provides detailed growth and phenotypic metrics of small molecules on Chlamydomonas [29].

\section{Results}

\section{Chlamydomonas as a chemical biology model}

To screen for small molecules that inhibit the growth of Chlamydomonas we performed an 80-hour fitness assay (Figure 1a). Our assay takes advantage of tools developed for yeast growth that monitor optical density (OD) of individual microtiter plate wells several times each hour, resulting in high resolution growth curves $[27,30]$. This high-throughput method combines a liquid handling robot, plate reader and integration software [30]. Growth was performed at constant temperature $\left(22^{\circ} \mathrm{C}\right)$, agitation $(150 \mathrm{rpm})$ and illuminance $\left(40 \mu \mathrm{mol}\right.$ photons $\left./ \mathrm{m}^{2} \mathrm{~s}\right)$ and was validated in both 96- and 384-well plates.

To determine conditions for small molecule screening on Chlamydomonas we performed growth assays with solid and liquid TAP media with varying concentrations of solvent (DMSO) or small molecules. DMSO is a preferred diluent because the majority of available chemicals are soluble in DMSO at high concentrations and many chemical libraries are pre-plated in DMSO. We tested CC-125, a common laboratory strain, in DMSO doses ranging from 0.25 to $2.5 \%$, in liquid and solid media to determine a dose for screening (Figure S1 in Additional file 1). In liquid TAP media DMSO concentrations above $1.0 \%(\mathrm{v} / \mathrm{v})$ had adverse effects, with cells collecting at the bottom of wells, and $2.5 \%$ severely reduced growth. On solid media DMSO was deleterious to growth at concentrations greater than $2.0 \%$, at which point cells became swollen and chlorotic. Based on these tests, we selected a concentration of $0.5 \%$ DMSO for liquid TAP and $1.0 \%$ for solid TAP media [16,31,32].

We next assayed the fitness of Chlamydomonas by light scattering $\left(\mathrm{OD}_{600}\right)$ in 96- and 384-well plates to determine the cell density that provided the most reproducible highresolution growth curves with the greatest dynamic range. Suitable volumes for each plate type were determined empirically, $200 \mu \mathrm{l}$ for 96 -well plates and $70 \mu \mathrm{l}$ for 384-well plates. In addition, we miniaturized Chlamydomonas fitness assays further by screening $9 \mu \mathrm{l}$ in 1,536-well plates (Figure S2 in Additional file 1).

In a pilot study, we screened 168 novel small molecules (plates 723N5803 and 723N5890 from the Chembridge Novacore collection [33]) at doses ranging from 25 to $200 \mu \mathrm{M}$ to determine a suitable screening dose. Virtually all cells died at concentrations above $100 \mu \mathrm{M}$ regardless of structure (data not shown). Compared to Saccharomyces cerevisiae, Chlamydomonas is significantly more sensitive to small molecules. We chose an initial screening dose of $25 \mu \mathrm{M}$ because it yielded the best balance of inhibition without being toxic. This dose is similar to that used in small-scale screens on Chlamydomonas, plants and zebrafish $[16,24,32,34,35]$.

Although we anticipated that the hydroxyproline-rich cell wall of Chlamydomonas could present a physical barrier for small molecule uptake [36]; based on the efficacy of our tests and those in the literature, Chlamydomonas is sufficiently permeable to diverse chemical structures. To directly evaluate small molecule permeability on Chlamydomonas, we quantified the intracellular accumulation of exogenously added chemicals using high-pressure liquid chromatography (HPLC). Briefly, Chlamydomonas cells were treated with select small molecules for 2 to 4 hours, washed and lysed, and lysate was separated via highpressure liquid chromatography (HPLC) [37]. We found that drugs accumulate in a dose-dependent manner, based on the appearance of a peak in treated samples that elutes at a similar time and with the same spectral qualities as the drug in buffer (Figure S3 in Additional file 1). Our results indicate that small molecule uptake and accumulation in Chlamydomonas is more efficient than in Caenorhabditis elegans and S. cerevisiae [37,38].

We tested the growth of wild-type strains, non-motile mutants, as well as mutants that display altered phototaxis or photosynthesis, in our automated fitness assay to compare growth curves among the different strains (Table S1 in Additional file 2). All strains tested show comparable growth dynamics compared to wild-type (CC-125); however, motility mutants, bld2 (CC-478) and pf14 (CC-1032), occasionally show jagged growth curves, suggesting that their lack of motility can cause cells to aggregate and/or pool at the bottom of wells, which could result in erratic OD readings from one time point to the next. To minimize these effects plates are shaken at $150 \mathrm{rpm}$ during growth.

\section{Chlamydomonas small molecule screens}

We screened C. reinhardtii (CC-125) for sensitivity to compounds against several commercially available chemical collections, including the 'FDA library', a collection of 640 approved and off market drugs (Enzo Life Sciences, Farmingdale, NY, USA); 'Tim Tec', a 280 member natural 
A

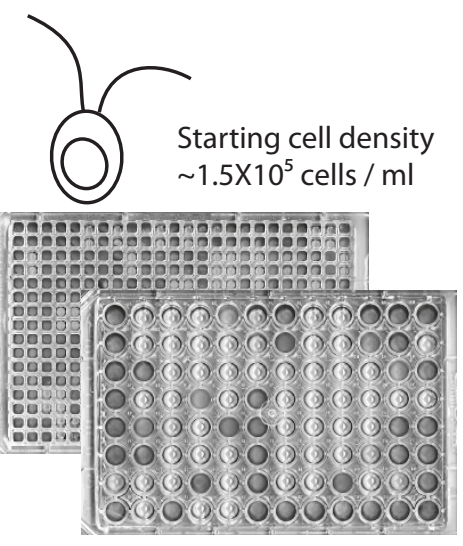

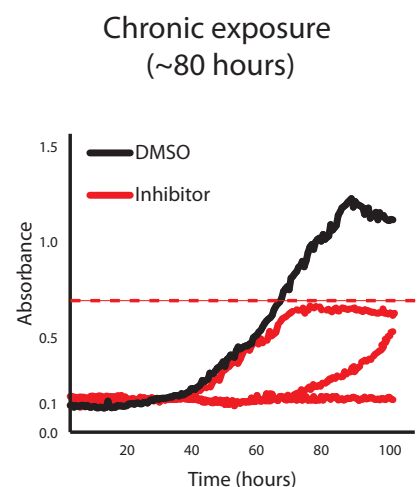

( $\sim 80$ hours)

B
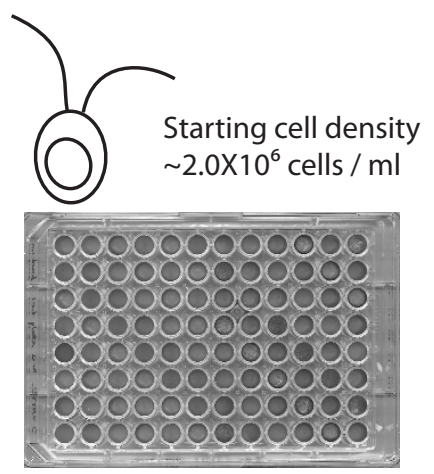

Acute exposure (4-8 hours)
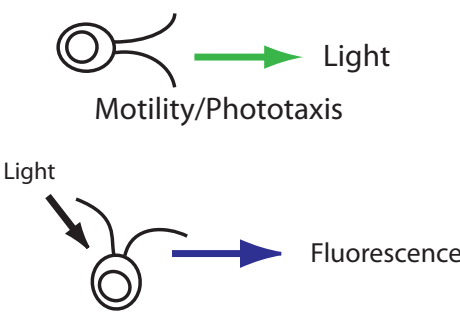

Photosynthesis
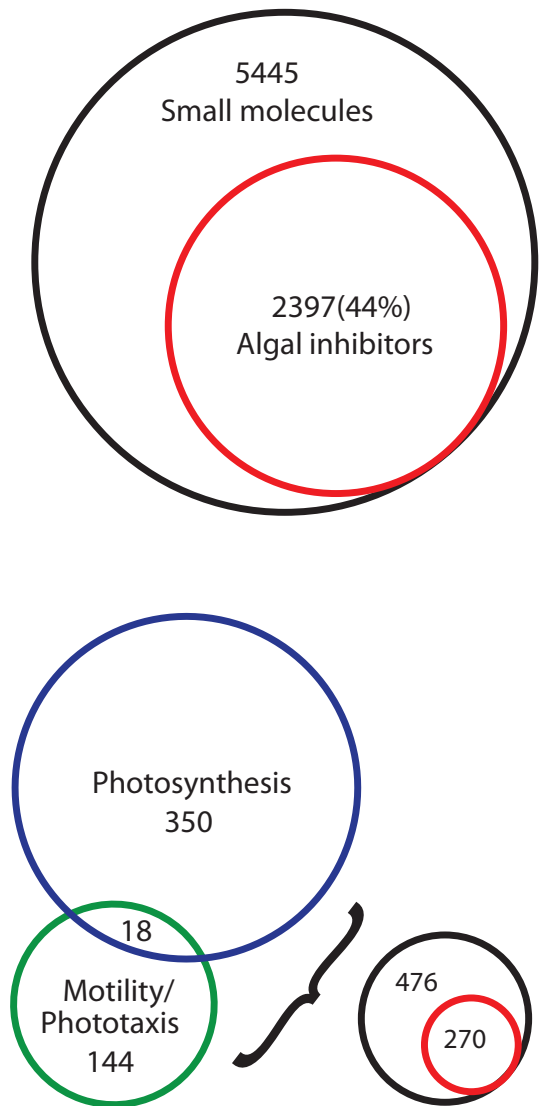

Figure 1 Chlamydomonas reinhardtii small molecule screens. (a) Chronic fitness screen: 96- and 384-well microtiter plates were inoculated with low density cells $\left(1.5 \times 10^{5}\right.$ cells $\left./ \mathrm{ml}\right)$ and small molecules were added to a final concentration of $25 \mu \mathrm{M}(0.5 \mu \mathrm{g} / \mathrm{ml} \mathrm{FDA} \mathrm{library)}$. Growth was monitored by optical density in our Freedom Evo robotics platform every 30 minutes, resulting in detailed growth curves. A total of 5,445 small molecules were screened with $44 \%$ inhibiting Chlamydomonas growth by $35 \%$ or more compared to in-plate controls. (b) Acute exposure screen: 96-well microtitre plates were prepared similarly to the chronic screen except with a higher cell density $\left(2 \times 10^{6}\right.$ cells $\left./ \mathrm{ml}\right)$. Plates were assayed for alterations in motility utilizing a phototaxis assay or photosynthesis by assaying fluorescent induction. Of the 476 unique acute inhibitors, $45 \%$ were also active in the chronic exposure screen.

product library consisting of herbicides and natural extracts (TimTec LLC, Newark, DE, USA); and the 'yactives library', a novel small molecule collection that we collated based on yeast growth inhibition from ChemBridge (NOVACore and DIVERSet) and ChemDiv (Divers) collections [38] (Table S2 in Additional file 2). In total we screened 5,445 unique small molecules in triplicate (with the exception of the TimTec library because of limited amounts and two NOVACore plates from the pilot screen). We calculated the standard deviation to assess variation between replicates and developed a compound activity score based on the average of the triplicate data. Actives were scored based on the area under each growth curve versus an in-plate DMSO control. Compounds that resulted in a growth ratio of 0.65 or less (that is, $35 \%$ inhibition), defined Chlamydomonas fitness inhibitors. Using this metric, $44 \%(2,397)$ of small molecules from our screen of 5,445 distinct small molecules altered algal growth (Figure 1a). This enrichment is quite high, and likely reflects that the majority of these small molecules have been pre-selected for bioactivity. Indeed, we have shown that compared to random compounds, the yactive library is enriched 3- to 12-fold when screened against Escherichia coli, whole worms and cultured mammalian cells [38].

To complement the fitness screen, we performed an acute exposure screen to measure light- and motilityrelated phenotypes. By treating Chlamydomonas for hours (as opposed to days) we can observe immediate effects of chemical activity on cells within a single cell cycle. We assayed motility/phototaxis and photosynthesis because they are well-studied phenotypes and are amenable to detailed downstream follow-up analysis (Figure 1b). These screens were performed similar to fitness screens, but with 
a higher starting cell density $\left(2 \times 10^{6}\right.$ cells $/ \mathrm{ml}$ versus $1.5 \times$ $10^{5}$ cells $/ \mathrm{ml}$ ) and for a shorter time ( 4 to 8 hours versus approximately 80 hours). Phototaxis was evaluated by Chlamydomonas' response to a strong directional light; 3 minutes following light exposure an image was captured and the response scored. We tested photosynthetic efficiency by assaying fluorescence induction at $685 \mathrm{~nm}$, the fluorescent maxima for photosystem II (PSII). Specifically, plates were transferred from low light into a plate reader, shaken at $100 \mathrm{rpm}$ in the dark for 20 seconds and then excited with $470 \mathrm{~nm}$ light, an optimal wavelength for photosynthetic function. In this procedure a higher fluorescence signal corresponds to reduced photosynthetic capacity, because the emitted light is released as fluorescence rather than being used for photosynthesis [39].

From the 5,445 chemicals screened in the chronic assay, 4,841 (88.9\%) were screened in the acute assay. In this latter set of compounds we identified 144 motility/ phototaxis modulators and 350 photosynthetic inhibitors, of which 18 were found in both assays (Figure 1b). Of these 476 small molecules active in these acute screens, 270 were also growth inhibitors, suggesting that their growth inhibition may be a result of modulating components involved in motility/phototaxis or photosynthesis, or alternatively, by secondary effects on essential cellular processes. Motility/phototaxis modulators were separated into two classes, 106 inhibitors of motility (no response to directional light) and 38 modulators of phototaxis sign (altered response to directional light). Vinpocetine, a phosphodiesterase and $\mathrm{Na}^{+}$channel inhibitor [40,41], is a phototaxis modulator resulting in positive phototaxis in our assay (untreated cells display negative phototaxis; Figure S4 in Additional file 1), which likely modulates phototaxis by altering concentrations of $\mathrm{Na}^{+}$and $\mathrm{Ca}^{2+}$ ions.

Following the acute and chronic exposure screens we pinned the cells in each well onto TAP agar without compound to determine which chemicals under each treatment were cytocidal or cytostatic. We performed the cytocidal/cytostatic assay for cells treated in the fitness screen (approximately 80 hours) and found that $11.5 \%$ are cytocidal and $33.2 \%$ are cytostatic. The difference in cytocidal/cytostatic percentages between the chronic and acute assays suggests that exposure time is critical, and that most small molecules under short-term exposure are reversible. However, we found that only $0.9 \%(44 / 4,841)$ of chemicals are cytocidal and $0.3 \%(17 / 4,841)$ are cytostatic under the short exposure of the acute screen (4 to 8 hours). The results from the acute screen indicate that fitness inhibitors also show effects in our acute exposure experiments and demonstrate that the fitness assay identifies inhibitors of specific, growth-dependent processes.

To gain insight into the physiochemical properties that confer activity on Chlamydomonas, we clustered all active small molecules by their structural similarity and mapped them on each phenotype (fitness, motility/ phototaxis, and photosynthesis) to define substructures enriched for specific activities. We calculated ECFP_4 similarity [42] for each small molecule versus the entire 5,445 screened collection and using Cytoscape [43] and a cutoff of 0.5 (on a scale of 0 to 1.0 , with 1.0 representing identical compounds), we clustered and visualized the data using a network topology (Figure 2). We then mapped our phenotypic data using Cytoscape onto our small molecule network to identify clusters that group according to structure and phenotype.

Several major structural classes of compounds are highlighted to illustrate that related structures cluster with specific phenotypes (Figure 2and Table 1). We highlighted five chemical clusters (groups I to IV) based on their phenotypic activity profile. Fitness inhibitors are by far the largest class of small molecule identified in our screens. Group I inhibitors, characterized by a shared benzylmethyl-phenylethyl-piperidinyl-methylamine, predominately inhibit growth. Group II compounds affect motility/ phototaxis, which appear to inhibit motility as a result of their high toxicity, as cells in the phototaxis assay show no response to directional light and most treated cells are lysed. We found that photosynthetic inhibitors are found evenly across our network, indicating that diverse chemical structures can alter photosynthesis. Not surprisingly, there is significant overlap between photosynthesis and growth inhibitors - for example, herbicides affect growth based on their effects on photosynthetic efficiency. Group III is the combination of two unrelated clusters, phenylpiperidine-oxazoles and benzoyl-piperidine, enriched for photosynthetic inhibitors. To assess the mode of action of these photosynthetic inhibitors, we screened a subset of the 350 photosynthetic inhibitors on a well-characterized DCMU resistant strain, which has the $\mathrm{V}>\mathrm{I}$ mutation in the D1 protein at residue 219 [44]. Based on the resistance of the mutant to uncharacterized photosynthesis inhibitors, we can gain insight into their mode of action and prioritize novel photosynthetic inhibitors for follow-up. Among 48 active photosynthetic inhibitors we found 23 chemicals that affect wild-type (CC-125) but not a DCMU-resistant line (CC-1403), suggesting the D1 mutation confers resistance to many compounds within our set of uncharacterized inhibitors. Group IV small molecules possess activity in all phenotypes tested, indicating the biphenyl-pyrazole substructure is particularly active. Interestingly, most chemicals that reverse Chlamydomonas response to light are from group IV.

\section{A structural model for Chlamydomonas bioactive compounds}

Using chemical fingerprinting analysis of the active versus inactive small molecules, we constructed a model to 


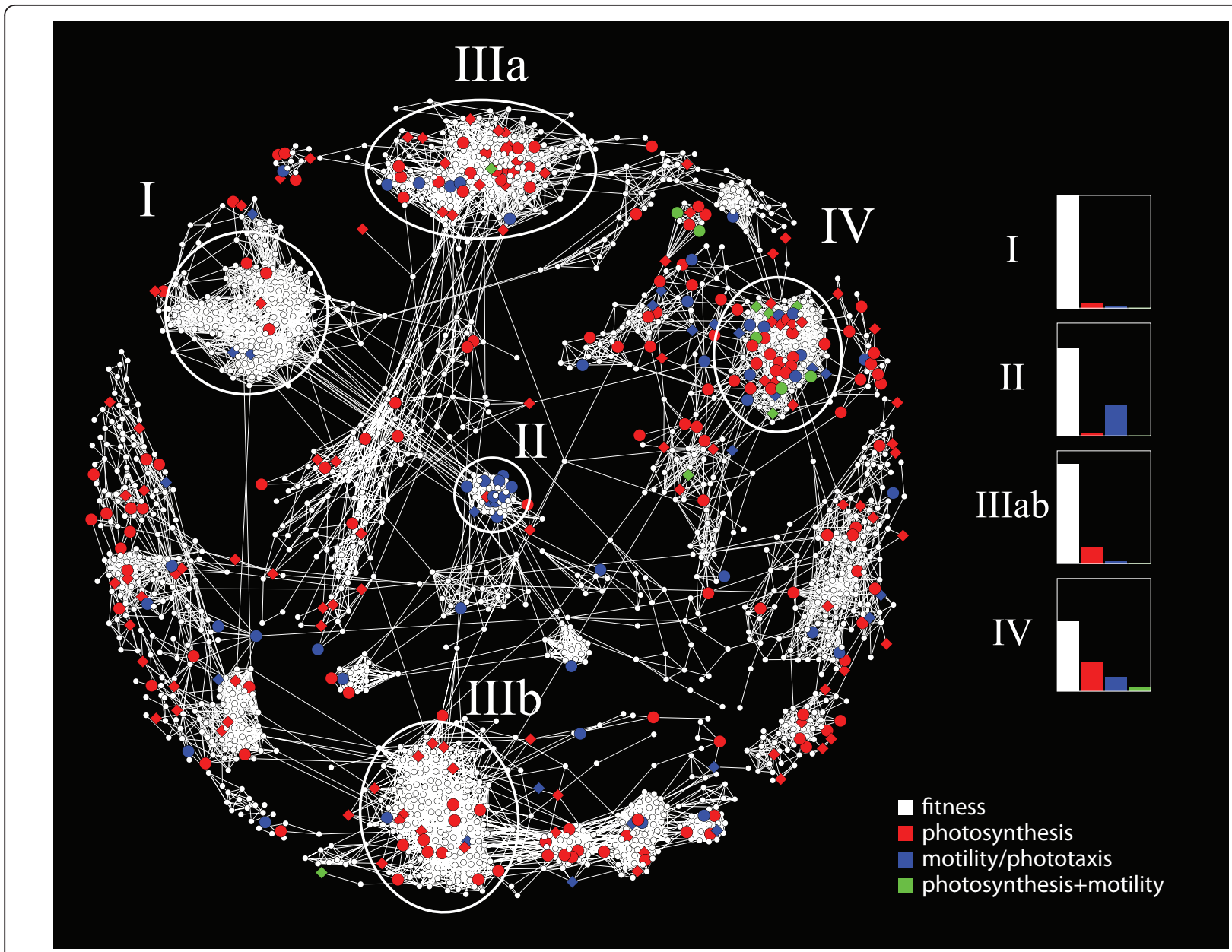

Figure 2 Network view of active molecules. All active small molecules were clustered according to their ECFP_4 chemical fingerprints. Each node represents a unique small molecule: 1,802 small molecules cluster together and are shown here. Edges represent structural relatedness at a cutoff of ECFP_4 greater than 0.5. Core structural clusters are outlined and designated groups I to IV. Acute screen data were mapped onto the active network with white fitness inhibitors, red photosynthetic inhibitors, blue motility/phototaxis modulators, and green photosynthetic and motility/phototaxis; circles indicate fitness inhibition, diamonds indicate no fitness inhibition. Bar graphs: phenotypes for each chemical group are displayed as a percentage.

Table 1 Groups I to IV core chemical structures and reported activities

\begin{tabular}{|c|c|c|c|}
\hline $\begin{array}{l}\text { Core } \\
\text { structure } \\
\text { group }\end{array}$ & Phenotype & PubChem bioassays (AID) & Named drugs and uses \\
\hline I & Fitness & $\begin{array}{l}\text { PubChem AID 317390, 317391, 317396, 317399, 317400, 5512, 52703; } \\
\text { displaces binding of sigma receptor, binds sigma receptor, binds 5HT-2A } \\
\text { receptor, inhibits chemokine receptor } 3 \text { (CCR3) }\end{array}$ & \\
\hline$\|$ & $\begin{array}{l}\text { Motility/ } \\
\text { Phototaxis }\end{array}$ & PubChem AID31792; acetocholinesterase inhibitor & Cyclizine, antihistamine; benethamine, \\
\hline Illa & Photosynthesis & $\begin{array}{l}\text { PubChem AID1865, 2314, 2315, 2546, 504333, 493056; epigenetic } \\
\text { regulator, shiga toxin inhibitor, inhibits retinoic acid related orphan } \\
\text { receptor gamma, inhibits BAZ2B, increases thyrotropin releasing } \\
\text { hormone receptor }\end{array}$ & $\begin{array}{l}\text { Darglitazone, muraglitazar, peroxisome } \\
\text { proliferation activated receptor gamma, } \\
\text { antiglycemic; oxaprozin, NSAID }\end{array}$ \\
\hline $111 \mathrm{~b}$ & Photosynthesis & $\begin{array}{l}\text { PubChem AID894, 1529, 145655, 5512; binds 5HT-2A receptor, inhibits } \\
\text { MEK5 kinase, inhibits 15-hydroxyprostaglandin dehydrogenase }\end{array}$ & Tolperisone, muscle relaxant \\
\hline IV & $\begin{array}{l}\text { Active in all } \\
\text { phenotypes }\end{array}$ & $\begin{array}{l}\text { PubChem AID265123, 265124, 265125, 265126, 265127; binds dopamine } \\
\text { receptors D2, D3, D4, D1A }\end{array}$ & $\begin{array}{l}\text { Fezolamine, antidepressant; lonazole, NSAID, } \\
\text { COX2 inhibitor }\end{array}$ \\
\hline
\end{tabular}


predict active algal inhibitors. Chemical structures of active and inactive small molecules were analyzed using the ECFP_4 chemical fingerprinting [42], and were used to train a naïve Bayes model to determine chemical groups over-represented in the active class. To test the performance of the model we trained it using four-fifths of the dataset and then predicted actives in the remaining fifth of the data. This was repeated five times to ensure that each fifth of the data was used as the comparison set. We then generated one model for each phenotype to predict compounds that were active in fitness, motility/ phototaxis, and photosynthetic structures. Using these models we ranked small molecules by predicted algal activity and compared the number of tested algal active compounds identified versus a randomly ordered set. At a predictive rate of $10 \%$ (selecting the top $10 \%$ of ranked compounds versus a random selection), the fitness, motility/phototaxis, and photosynthetic models showed enrichment for actives of $1.6 \times, 3.8 \times$, and $2.8 \times$, respectively (Figure $3 \mathrm{a}-\mathrm{c}$ ). These enrichments could easily save significant costs in labor and time (for example, screening $10 \%$ of a $10 \mathrm{~K}$ library with a hit rate of $44 \%$ results in 440 hits randomly versus 704 hits from a prioritized list with an enrichment of $1.6 \times$ ). We next used our model to predict algal actives on a 50,000-member small molecule library (NOVACore, ChemBridge, San Diego, CA, USA) and empirically tested 253 of the predicted compounds. Our model accurately predicted active small molecules in the unscreened library, enriching for algal fitness inhibitors $2.8 \times$ over a non-prioritized set of the same library, demonstrating the utility of such predictive models for prioritizing molecules for screening.

\section{Human antipsychotics alter Chlamydomonas motility}

Our screen included two small molecule collections of known inhibitors, the 'FDA and TimTec' libraries, of which approximately 7\% (61) are algal actives. These compounds were enriched for chloroplast and mitochondria targets, and antifungals including ergosterol inhibitors, and human antipsychotics. The antipsychotics were enriched for those involved in dopamine and serotonin signaling. Intriguingly, we found that a majority of our chemical groups (Figure 2) have activity in diverse dopamine and serotonin assays (Table 1).

To better understand the activity of antipsychotics on Chlamydomonas we focused on the atypical antipsychotic dibenzazepines because of their activity in our screens, therapeutic relevance and availability of structural analogs. In our dataset this cluster is composed of structural analogs of clozapine, including clothiapine (Figure 4a-c), an active algal growth inhibitor in our assay, and fluperlapine, which we found caused cell pooling in the acute exposure screen but does not affect fitness at screening doses.
Interestingly, clozapine, which differs from fluperlapine by one atom, does not show growth inhibition at the same concentration. When a broad range of concentrations was tested, clozapine and other analogs, loxapine, quetiapine, and clothiapine, produced a similar aggregation phenotype as fluperlapine and were all inhibitory at high concentrations, suggestive of different potencies but similar modes of action.

To further characterize the dibenzazepine effect, cells following fluperlapine treatment were observed microscopically (Figure 4d, e). Two predominant classes of cells were observed; large transparent cells (36/80) and small clusters of typically four cells (31/80). The large cells were chlorotic with vacuolated chloroplasts, indicating these cells were dead or dying. The cells within the four-cell clusters appeared normal, but remained encased in a parental cell wall. Mechanical stress released four aflagellate cells, indicative of a hatching defect (Figure 4f). Because hatching requires the cell wall degrading enzyme sporangin, which is expressed only in the flagella [45], aflagellate cells (that is, bld-2) have difficulty hatching [45]. Given the phenotypic similarities between fluperlapine treatment and flagellar mutations, we speculate that fluperlapine and its analogs could modulate flagellar growth/development to prevent hatching.

To determine if fluperlapine affects flagellar growth, we performed a flagellar regeneration assay in the presence of fluperlapine. Several inhibitors are known to shorten flagella, including IBMX, colchicine and cytochalasin D $[46,47]$. We deflagellated cells using the $\mathrm{pH}$ shock method [48] and assayed flagellar length every 20 to 30 minutes (Figure 4g) in the presence of $100 \mu \mathrm{M}$ fluperlapine. This dose resulted in an observable effect, yet did not affect viability after washout. Over the course of approximately 2 hours, flagella regenerated to near pre-deflagellation lengths $(8.97 \pm 0.5 \mu \mathrm{m}$; pre-deflagellation $10.9 \pm 0.7 \mu \mathrm{m})$ in solvent-treated cells, while fluperlapine-treated cells regenerated to just over half length $(7.0 \pm 0.5 \mu \mathrm{m})$. We also treated cells with several doses of fluperlapine and clozapine for 12 hours. Fluperlapine-treated cells $(50 \mu \mathrm{M})$ had flagella $(7.2 \pm 0.4 \mu \mathrm{m})$, similar to that observed in the flagellar regeneration assay; however, cells treated with clozapine (50 and $100 \mu \mathrm{M})$ and high concentrations of fluperlapine $(100 \mu \mathrm{M})$ were aflagellate and inviable.

\section{Discussion}

In this study we demonstrate that high-throughput screening of the model alga $C$. reinhardtii is an effective means to identify novel small molecule probes. Our work builds on previous studies focused on small molecule herbicides and well-characterized inhibitors and combines it with highthroughput methods developed for bacteria and yeast. For example, the PSII inhibitors DCMU and atrazine, and 

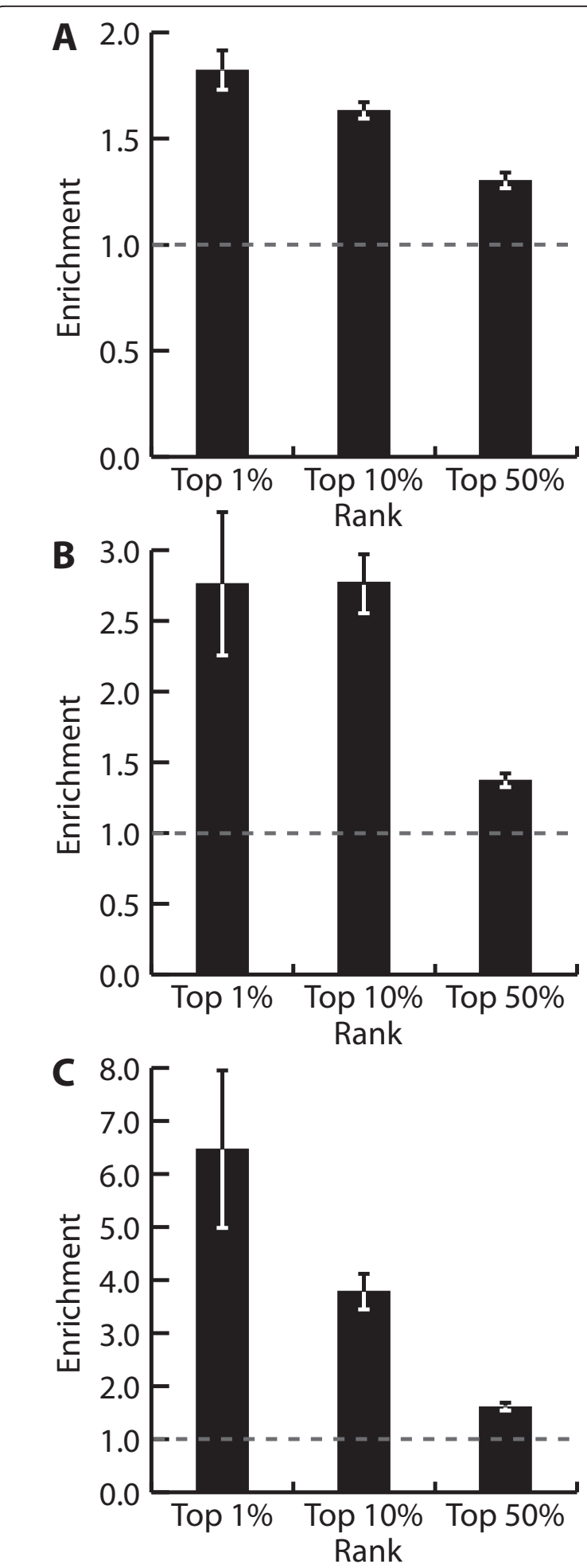

Figure 3 Naïve Bayes modeling for active small molecule prediction. (a) Fitness model. (b) Photosynthesis model. (c) Motility/phototaxis model. (a-c) The model was trained on four-fifths of the active set and tested against the remaining fifth. Assessment is repeated five times and compared to random selection of active small molecules to calculate the model enrichment factor. Error bars represent standard error of five replicates. other modulators of photosynthesis, have contributed to our understanding of photosynthesis and signaling [44,49]. Antibiotics that target the chloroplast were instrumental in demonstrating uniparental chloroplast inheritance [6]. Research on motility and the cell cycle sparked interest in various classes of modulating compounds, including microtubule inhibitors [50,51], many of which can discriminate between animal and plant tubulins and are therefore useful in agriculture [52]. Inhibitor studies have also shed light on the biology of flagellar regeneration [46] and microtubule organization [31]. Chemical interrogation of phototaxis using chemical screens has identified modulators of motility and light perception $[24,53,54]$. In addition, studies on a diverse group of chemical classes, including anesthetics [26,46,55,56], phosphodiesterase inhibitors [23,57], DNA damaging drugs [58], antipsychotics [22], antifungals and translation inhibitors $[55,56,59,60]$, have established the sensitivity of Chlamydomonas to diverse chemical classes and structures. Recent efforts have exploited Chlamydomonas' small molecule sensitivity to develop it as a biomonitor of pesticide, herbicide and heavy metal accumulation in water systems $[19,61]$.

Our screens are quantitative (providing high-resolution growth curves for each small molecule) and large enough to identify active compounds that can be classified into phenotypic categories. Profiling screens incorporating fitness readouts have shown remarkable predictive ability in binning known inhibitors with small molecule libraries [28,38,62-64].

Using the results from our screens, we generated a model that effectively predicts the Chlamydomonas bioactivity of compounds and allows one to rank and prioritize libraries for screening. By training a Naïve Bayes model with chemical fingerprinting data from algal actives we were able to predict fitness inhibitors in an unscreened small molecule library, resulting in enrichment of actives identified by $2.8 \times$. Our model can be improved in an iterative manner, as more data become available for training, increasing its predictive accuracy.

All screens are accessible for individual query or bulk download from our website [29]. This website contains fitness and phenotypic metrics for each small molecule tested, as well as links to several chemical repositories, including PubChem [65], ChemBank [66] and PharmGKB [67]. Also included are several unscreened chemical libraries with predicted activity scores on Chlamydomonas as a resource for further screening.

Finally, we identified a class of neuroleptics, clozapine analogs that appear to modulate Chlamydomonas flagellar growth/function. In animals, these drugs target serotonin and dopamine receptors; however, such targets are absent in green algae and therefore the pathways affected in Chlamydomonas may represent functions that are evolutionarily 


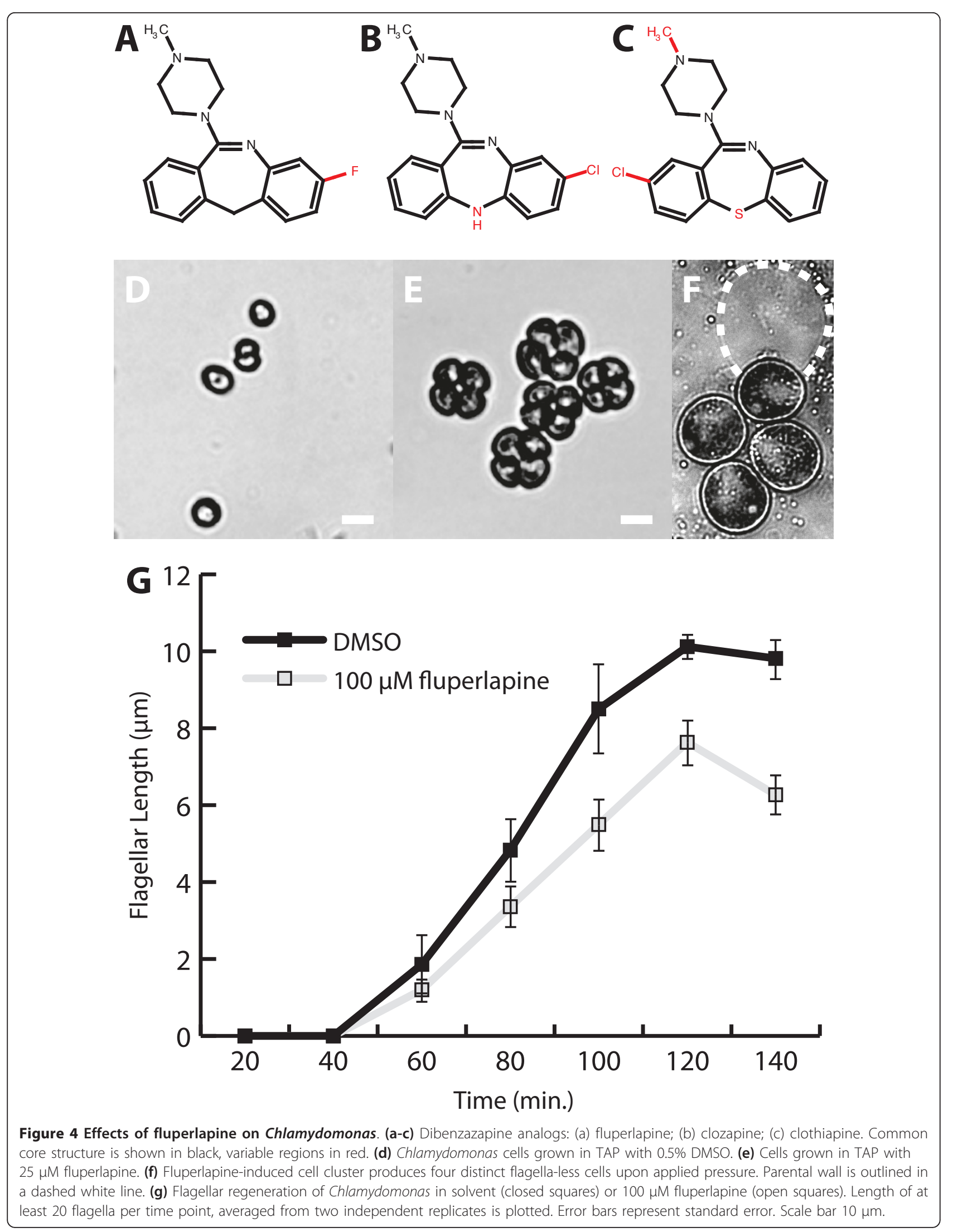


conserved and ancient. For example, Avasthi et al. [22] recently reported results from a Chlamydomonas motility screen using the chemical library LOPAC (Library of Pharmacologically Active Compounds), in which they found an enrichment in antipsychotics with reported targeting to G-protein coupled receptors and observed modulation of mammalian ciliary length. Our findings on fluperlapinetreated cells suggest modulation of flagellar growth based on the absence of flagella in chronically grown cells, aberrant hatching and reduced flagellar regeneration.

\section{Conclusions}

The maturation of Chlamydomonas as a model 'plant' makes its description as the green yeast more appropriate now than ever before. Chlamydomonas genomics, combined with the features that have made it an excellent genetic system, promise applications beyond the laboratory - for example, in biofuel development. A recent observation shows that changes in culture conditions can coax Chlamydomonas cells to produce an abundance of triacylglycerol, to the point where it comprises much of the cell volume (reviewed in [68]). The platform we describe here is well suited to (i) systematize molecular breeding experiments and (ii) explore chemical and environmental space to uncover perturbations that produce a desired phenotype. Combining such high-throughput screening capacity with genetic perturbations across the Chlamydomonas genome will permit new research insights for understanding 'nonyeast' biology - for example, ciliopathies, photosynthesis, and biofuel precursor production, to name a few. Indeed, recent advances in next-generation sequencing for assessing genome-wide mutant collections (reviewed in [69]) make the development of a systematic Chlamydomonas mutant collection (for example, using barcoded transposons or complete deletions $[70,71])$ not only possible, but essential.

\section{Materials and methods}

\section{Strains and growth conditions}

Screens were performed using the Chamydomonas reference strain CC-125 (nit1- nit2- a gift from the Dutcher Lab) grown in TAP media [72] under constant illumination (LED strip light, Lumicrest, Toronto, ON, Canada). Cells for screening were inoculated from pre-cultures in which $4 \mathrm{ml}$ of TAP was inoculated from a single colony, cells were cultured to $\mathrm{OD}_{600} \sim 0.1$ for chronic or $\mathrm{OD}_{600} \sim 0.4$ for acute screens.

Growth assays were performed in clear, flat-bottom 96- and 384-well microtiter plates (VWR International, Mississauga, ON, USA) sealed with adhesive plate seals (catalogue number AB-0580) using a custom developed platform incorporating microtitre plate reader Safire2 and the Freedom EVO (Tecan-US, Durham, NC, USA). Chronic screens were carried out at an initial cell density of $1.5 \times$ $10^{5} \mathrm{cells} / \mathrm{ml}\left(\mathrm{OD}_{600} \sim 0.1\right)$. A final screen concentration of $25 \mu \mathrm{M}(10 \mu \mathrm{g} / \mathrm{ml}$ FDA library $)$ with a DMSO solvent concentration at $0.5 \%$ in a total volume of 190 to $200 \mu \mathrm{l} /$ well was chosen based on initial testing. We found that concentrations of DMSO greater that $0.5 \%$, in liquid assays, resulted in aberrant growth (aggregating cells; Figure S1 in Additional file 1). Plates were shaken in constant light to saturation at $150 \mathrm{rpm}$, for 3 to 4 days, with at least four DMSO controls per plate. Algal growth was assayed using a Tecan Safire2 plate reader measuring OD every $30 \mathrm{~min}$ utes at $\mathrm{OD}_{600} \mathrm{~nm}$. OD readings are output to provide detailed growth curves (OD over time) that can distinguish percent variations in growth. We were also able to assess fitness in 1,536-well plates as above. Plates were prepared by inoculating $50 \mu \mathrm{l}$ of cells with drug or solvent to the appropriate concentration, aliquoting $9 \mu \mathrm{l}$ to each well, and spinning down at $500 \mathrm{rpm}$ for $10 \mathrm{~s}$ to remove bubbles.

Acute screens were prepared as above except they were performed only in 96-well microtiter plates at an initial cell density of $2.0 \times 10^{6}$ cells $/ \mathrm{ml}\left(\mathrm{OD}_{600} \sim 0.4\right)$ and incubated between 4 and 8 hours. Phototaxis response was assayed by placing 96 -well plates in a strong directional light for 3 minutes. Images for each plate were captured and the assay was repeated at least 15 minutes later on the other side of the plate to improve resolution of wells far from the light. Images were scored for inhibitors of motility, no movement, or movement towards light, the opposite of untreated cells. Photosynthetic efficiency was assayed by fluorescent induction in the Safire2 plate reader. Plates were kept in low light prior to the assay. We assessed photosynthetic efficiency by exciting with $470 \mathrm{~nm}$ actinic light and measuring the emission at 680 $\mathrm{nm}$, in which a higher reading reports inhibited photosynthesis. To determine cytotoxicity (cytocidal/cytostatic) we pinned treated cells onto $2 \%$ agar in 96 -well format and allowed them to grow for 10 days at $24^{\circ} \mathrm{C}$ before they were analyzed.

\section{Chemicals and libraries}

The chemical libraries screened were FDA BML-640 (Enzo Life Sciences, Farmingdale, NY, USA), Yactives, a prescreened yeast actives set derived from ChemBridge (NOVACore and DIVERSet, San Diego, CA, USA) and ChemDiv (Divers, San Diego, CA, USA), and two stock plates from ChemBridge (NOVACore, San Diego, CA, USA). TimTec NPL-280 (TimTec LLC, Newark, DE, USA) was supplied at $5.0 \mathrm{mM}$ in DMSO and was a gift from D Desveaux (University of Toronto). Lugol's stain was obtained from Sigma (62650-100ML-F, St Louis, $\mathrm{MO}, \mathrm{USA})$ and diluted to $10 \mu \mathrm{M}$ in water. Formaldehyde 10\% (04018, Polysciences, Warrington, PA, USA) was diluted to $1 \%$ in water. Fluperlapine was ordered 
from Enzo Life Sciences (BML-NS109, Farmingdale, NY, USA) and suspended in DMSO to a stock concentration of $100 \mathrm{mM}$.

\section{Screening chemical libraries on $C$. reinhardtii}

A compound was considered active on $C$. reinhardtii if the area under the growth curve after reaching saturation was less than $65 \%$ of the DMSO control (ratio [compound/control] $<0.65$ ). Automatic flagging of actives was confirmed by visual inspection of the data. Compounds were added to the culture using a $2 \mu \mathrm{l}$ or $600 \mathrm{nl}$ pin tool (V\&P Scientific, San Diego, CA, USA) for 96- or 384-well microplates, respectively, to dilute the compounds 200 times to a final DMSO concentration of $0.5 \%$.

\section{Cheminformatic analysis}

For all chemicals ECFP_4 similarity was calculated using the cheminformatic package in Pipeline Pilot version 6.1 (Scitegic Inc. Accelyrs, San Diego, CA, USA). Chemicals with a similarity of greater than 0.5 (1.0 being identical) were visualized using Cytoscape version 2.8.2; results from phenotypic screens were added as attributes and were used to alter node shape and color. Marvin version 5.4.1 (ChemAxon, Budapest, Hungary) was used for drawing and displaying chemical structures. Naïve Bayes model building was performed as described [38].

\section{Flagellar regeneration}

Cells were grown for two days in a $16 \mathrm{~h} / 8 \mathrm{~h}$ light/dark cycle to an $\mathrm{OD}_{600} \sim 0.4$. Cells were deflagellated using the $\mathrm{pH}$ shock method [48] by adding $350 \mu \mathrm{l}$ of $0.5 \mathrm{~N}$ acetic acid, inverted for 40 seconds, and neutralized with $125 \mu \mathrm{l}$ $\mathrm{Na} 2 \mathrm{CO} 3$, to $6 \mathrm{ml}$ of culture. Deflagellation was confirmed by observation of cells at $40 \times$ using a DMIL inverted light microscope (Leica). Deflagellated cells were aliquoted into $1.5 \mathrm{ml}$ tubes and drug or solvent was added. Flagella were observed every 20 to 30 minutes by fixing cells with $6.67 \times$ $10^{-3} \%$ formaldehyde, a concentration that preserved flagella during the 20-minute observation period. Flagella were observed with the $100 \times$ objective and acquired using AxioVision software on an Axiovert $200 \mathrm{M}$ microscope (Carl Zeiss).

\section{HPLC analysis}

A $100 \mathrm{ml}$ culture was grown for four days, spun down and resuspended in $20 \mathrm{ml}$ fresh TAP. Aliquots $(1 \mathrm{ml})$ were treated with drug or solvent for $3 \mathrm{~h}$. Cells were then washed three times with TAP, resuspended in $50 \mu \mathrm{l}$ TAP media, transferred to clean $1.5 \mathrm{ml}$ tubes and stored frozen at $-20^{\circ} \mathrm{C}$. The samples were later lysed with $50 \mu \mathrm{l}$ SDS-EB buffer (2\% SDS, $400 \mathrm{mM} \mathrm{NaCl}, 40 \mathrm{mM}$ EDTA, $100 \mathrm{mM}$ Tris- $\mathrm{HCl}, \mathrm{pH}$ 8.0) and incubated at $60^{\circ} \mathrm{C}$ for $1 \mathrm{~h}$. Samples were frozen at $-80^{\circ} \mathrm{C}$ and later processed on HPLC as described [37].

\section{Additional material}

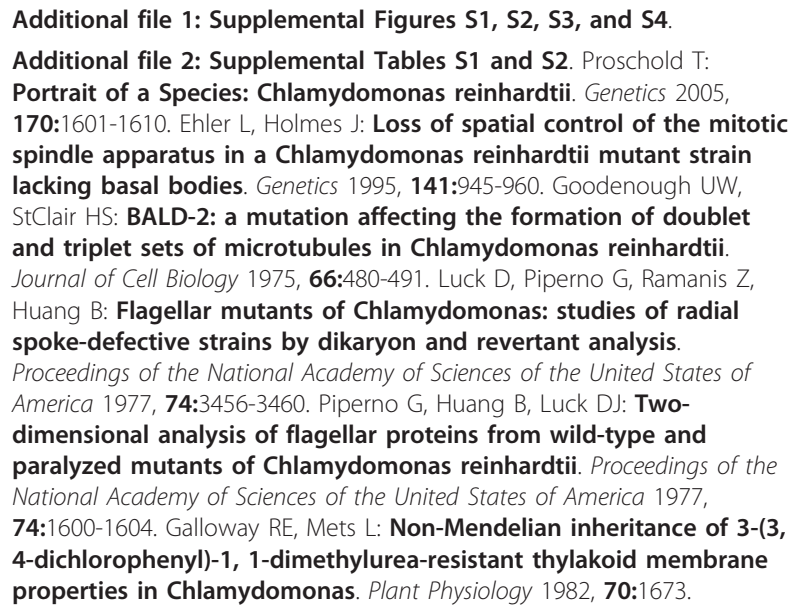

\section{Abbreviations}

DMSO: dimethyl sulfoxide; HPLC: high-pressure liquid chromatography; OD: optical density.

\section{Authors' contributions}

SEA carried out the study. CL performed screening and deflagellation assays. $\mathrm{KL}$ performed screening and motility/phototaxis assays. AM performed photosynthesis retests and HPLC assays. AS and IW performed cheminformatic analysis and web development. MU set up initial experiments and robotics. MP assisted with data collection and developed YG software. SEA and CN conceived of the study and wrote the manuscript. All authors read and approved the manuscript.

\section{Competing interests}

The authors declare that they have no competing interests.

\section{Acknowledgements}

We thank Susan Dutcher for providing strains, CC-125, and bld2, and for advice on Chlamydomonas growth and experimental design. Thanks to Graham Bell for advice and wild-type strains. And thank you to Andrew Burns for running HPLC samples. CN and GG are supported by a grant from the NHGRI and the Canadian Cancer Society (020380).

\section{Author details}

'Donnelly Centre for Cellular and Biomolecular Research, University of Toronto, 160 College Street, Toronto, Ontario M5S 3E1, Canada. ${ }^{2}$ Banting and Best Department of Medical Research, University of Toronto, 112 College Street, Toronto, Ontario M5G 1L6, Canada. ${ }^{3}$ Department of Molecular Genetics, University of Toronto, 1 King's College Circle, Toronto, Ontario, M5A 1A8, Canada. ${ }^{4}$ Department of Pharmaceutical Sciences, University of Toronto, 144 College Street, Toronto, Ontario M5S 3M2, Canada. ${ }^{5}$ Stanford Genome Technology Center, Palo Alto, CA 94304, USA. ${ }^{6}$ Novartis, 250 Massachusetts Ave., Cambridge 02139, USA.

Received: 1 August 2012 Accepted: 18 November 2012 Published: 18 November 2012

\section{References}

1. Mayer TU: Chemical genetics: tailoring tools for cell biology. Trends in Cell Biology 2003, 13:270-277.

2. McCourt P, Desveaux D: Plant chemical genetics. The New Phytologist 2010, 185:15-26.

3. Goodenough UW: Green yeast. Cell 1992, 70:533.

4. Merchant SS, Prochnik SE, Vallon O, Harris EH, Karpowicz SJ, Witman GB, Terry A, Salamov A, Fritz-Laylin LK, Maréchal-Drouard L, Marshall WF, Qu L-H, Nelson DR, Sanderfoot AA, Spalding MH, Kapitonov W, Ren Q, 
Ferris P, Lindquist E, Shapiro H, Lucas SM, Grimwood J, Schmutz J, Cardol P, Cerutti H, Chanfreau G, Chen C-L, Cognat V, Croft MT, Dent R, et al: The Chlamydomonas genome reveals the evolution of key animal and plant functions. Science 2007, 318:245-250.

5. Rochaix JD: Chlamydomonas reinhardtii as the photosynthetic yeast. Annual Review of Genetics 1995, 29:209-230.

6. Grant DM, Gillham NW, Boynton JE: Inheritance of chloroplast DNA in Chlamydomonas reinhardtii. Proceedings of the National Academy of Sciences of the United States of America 1980, 77:6067-6071.

7. Sager R, Ishida M: Chloroplast DNA in Chlamydomonas. Proceedings of the National Academy of Sciences of the United States of America 1963, 50:725.

8. Kato HE, Zhang F, Yizhar O, Ramakrishnan C, Nishizawa T, Hirata K, Ito J, Aita Y, Tsukazaki T, Hayashi S, Hegemann P, Maturana AD, Ishitani R, Deisseroth K, Nureki O: Crystal structure of the channelrhodopsin lightgated cation channel. Nature 2012, 482:369-374.

9. Foster KW, Saranak J, Patel N, Zarilli G, Okabe M, Kline T, Nakanishi K: A rhodopsin is the functional photoreceptor for phototaxis in the unicellular eukaryote Chlamydomonas. Nature 1984, 311:756-759.

10. Collins S, Bell G: Phenotypic consequences of 1,000 generations of selection at elevated $\mathrm{CO} 2$ in a green alga. Nature 2004, 431:566-569.

11. Ferris P, Olson BJSC, De Hoff PL, Douglass S, Casero D, Prochnik S, Geng S, Rai R, Grimwood J, Schmutz J, Nishii I, Hamaji T, Nozaki H, Pellegrini M, Umen JG: Evolution of an Expanded Sex-Determining Locus in Volvox. Science 2010, 328:351-354.

12. Lee $\mathrm{J}-\mathrm{H}$, Lin $\mathrm{H}$, Joo S, Goodenough U: Early sexual origins of homeoprotein heterodimerization and evolution of the plant KNOX/ BELL family. Cell 2008, 133:829-840

13. Renaut S, Replansky T, Heppleston A, Bell G: The ecology and genetics of fitness in Chlamydomonas. XIII. Fitness of long-term sexual and asexual populations in benign environments. Evolution 2006, 60:2272-2279.

14. Kozminski KG, Johnson KA, Forscher P, Rosenbaum JL: A motility in the eukaryotic flagellum unrelated to flagellar beating. Proceedings of the National Academy of Sciences of the United States of America 1993, 90:5519-5523.

15. Pazour G, Dickert B, Vucica Y, Seeley E, Rosenbaum J, Witman G, Cole D: Chlamydomonas IFT88 and its mouse homologue, polycystic kidney disease gene Tg737, are required for assembly of cilia and flagella. Journal of Cell Biology 2000, 151:709-718.

16. Hess F: A Chlamydomonas Algal Bioassay for Detecting Growth Inhibitor Herbicides. Weed Science 1980, 28:515-520.

17. Ma J, Xu L, Wang S: A quick, simple, and accurate method of screening herbicide activity using green algae cell suspension cultures. Weed Science 2002, 50:555-559.

18. Nagel K, Voigt J: In Vitro Evolution and Preliminary Characterization of a Cadmium-Resistant Population of Chlamydomonas reinhardtii. Applied and Environmental Microbiology 1989, 55:526-528.

19. Zhang S, Qiu CB, Zhou Y, Jin ZP, Yang H: Bioaccumulation and degradation of pesticide fluroxypyr are associated with toxic tolerance in green alga Chlamydomonas reinhardtii. Ecotoxicology 2011, 20:337-347.

20. Maucourt K, Agarwal M, René B, Fermandjian S: Use of Chlamydomonas reinhardtii mutants for anticancer drug screening. Biochemical Pharmacology 2002, 64:1125-1131.

21. Sena DW, Kulacki KJ, Chaloner DT, Lamberti GA: The role of the cell wall in the toxicity of ionic liquids to the alga Chlamydomonas reinhardtii. Green Chemistry 2010, 12:1066-1071.

22. Avasthi P, Marley A, Lin H, Gregori-Puigjane E, Shoichet BK, von Zastrow M, Marshall WF: A chemical screen identifies class a g-protein coupled receptors as regulators of cilia. ACS Chemical Biology 2012, 7:911-919.

23. Marshall WF: Quantitative high-throughput assays for flagella-based motility in chlamydomonas using plate-well image analysis and transmission correlation spectroscopy. Journal of Biomolecular Screening 2009, 14:133-141

24. Evans SK, Pearce AA, Ibezim PK, Primm TP, Gaillard AR: Select Acetophenones Modulate Flagellar Motility in Chlamydomonas. Chemical Biology \& Drug Design 2010, 75:333-337.

25. Lewin RA, Lee TH, Fang LS: Effects of various agents on flagellar activity, flagellar autotomy and cell viability in four species of Chlamydomonas (chlorophyta: volvocales). Symposia of the Society for Experimental Biology 1982, 35:421-437.

26. Nishikawa A, Sakamoto Y, Sakatoku A, Noguchi M, Tanaka D, Nakamura S: Induction of deflagellation by various local anesthetics in
Chlamydomonas reinhardtii Dangeard (Chlamydomonadales, Chlorophyceae). Phycological Research 2010, 58:79-87.

27. Giaever G, Flaherty P, Kumm J, Proctor M, Nislow C, Jaramillo DF, Chu AM, Jordan Ml, Arkin AP, Davis RW: Chemogenomic profiling: identifying the functional interactions of small molecules in yeast. Proceedings of the National Academy of Sciences of the United States of America 2004, 101:793-798.

28. Hillenmeyer ME, Fung E, Wildenhain J, Pierce SE, Hoon S, Lee W, Proctor M, St Onge RP, Tyers M, Koller D, Altman RB, Davis RW, Nislow C, Giaever G: The chemical genomic portrait of yeast: uncovering a phenotype for all genes. Science 2008, 320:362-365.

29. Chlamy Chem: small moleclue screen data for Chlamydomonas reinhardtii. [http://chlamychem.utoronto.ca]

30. Proctor M, Urbanus ML, Fung EL, Jaramillo DF, Davis RW, Nislow C, Giaever G: The automated cell: compound and environment screening system (ACCESS) for chemogenomic screening. Methods in Molecular Biology 2011, 759:239-269.

31. Ehler LL, Dutcher SK: Pharmacological and genetic evidence for a role of rootlet and phycoplast microtubules in the positioning and assembly of cleavage furrows in Chlamydomonas reinhardtii. Cell Motility and the Cytoskeleton 1998, 40:193-207.

32. James SW, Silflow CD, Stroom P, Lefebvre PA: A mutation in the alpha 1-tubulin gene of Chlamydomonas reinhardtii confers resistance to antimicrotubule herbicides. Journal of Cell Science 1993, 106:209-218.

33. Chembridge. [http://www.hit2lead.com]

34. Murphey RD, Stern HM, Straub CT, Zon LI: A chemical genetic screen for cell cycle inhibitors in zebrafish embryos. Chemical Biology \& Drug Design 2006, 68:213-219.

35. Zhao Y, Chow TF, Puckrin RS, Alfred SE, Korir AK, Larive CK, Cutler SR: Chemical genetic interrogation of natural variation uncovers a molecule that is glycoactivated. Nature Chemical Biology 2007, 3:716-721.

36. Woessner J, Goodenough UW: Volvocine cell walls and their constituent glycoproteins: An evolutionary perspective. Protoplasma 1994, 181:245-258.

37. Burns AR, Wallace IM, Wildenhain J, Tyers M, Giaever G, Bader GD, Nislow C, Cutler SR, Roy PJ: A predictive model for drug bioaccumulation and bioactivity in Caenorhabditis elegans. Nature Chemical Biology 2010, 6:549-557.

38. Wallace IM, Urbanus ML, Luciani GM, Burns AR, Han MKL, Wang H, Arora K, Heisler LE, Proctor M, St Onge RP, Roemer T, Roy PJ, Cummins CL, Bader GD, Nislow C, Giaever G: Compound prioritization methods increase rates of chemical probe discovery in model organisms. Chemistry \& Biology 2011, 18:1273-1283.

39. Bennoun P, Levine RP: Detecting mutants that have impaired photosynthesis by their increased level of fluorescence. Plant Physiology 1967, 42:1284-1287.

40. Hagiwara M, Endo T, Hidaka H: Effects of vinpocetine on cyclic nucleotide metabolism in vascular smooth muscle. Biochemical Pharmacology 1984, 33:453-457.

41. Sitges $M$, Nekrassov V: Vinpocetine selectively inhibits neurotransmitter release triggered by sodium channel activation. Neurochemical Research 1999, 24:1585-1591.

42. Rogers $D$, Brown RD, Hahn M: Using extended-connectivity fingerprints with Laplacian-modified Bayesian analysis in high-throughput screening follow-up. Journal of Biomolecular Screening 2005, 10:682-686.

43. Yeung N, Cline MS, Kuchinsky A, Smoot ME, Bader GD: Exploring biological networks with Cytoscape software. Current Protocols in Bioinformatics 2008, Chapter 8, Unit 813.

44. Erickson JM, Rahire M, Rochaix JD, Mets L: Herbicide resistance and crossresistance: changes at three distinct sites in the herbicide-binding protein. Science 1985, 228:204-207.

45. Kubo T, Kaida S, Abe J, Saito T, Fukuzawa H, Matsuda Y: The Chlamydomonas hatching enzyme, sporangin, is expressed in specific phases of the cell cycle and is localized to the flagella of daughter cells within the sporangial cell wall. Plant and Cell Physiology 2009, 50:572-583.

46. Dentler WL, Adams C: Flagellar microtubule dynamics in Chlamydomonas: cytochalasin D induces periods of microtubule shortening and elongation; and colchicine induces disassembly of the distal, but not proximal, half of the flagellum. Journal of Cell Biology 1992, 117:1289-1298.

47. Lefebvre P: Increased levels of mRNAs for tubulin and other flagellar proteins after amputation or shortening of Chlamydomonas Flagella. Cell 1980, 20:469-477. 
48. Witman GB, Carlson K, Berliner J, Rosenbaum JL: Chlamydomonas flagella. I. Isolation and electrophoretic analysis of microtubules, matrix, membranes, and mastigonemes. Journal of Cell Biology 1972, 54:507-539.

49. Erickson JM, Rahire M, Bennoun P, Delepelaire P, Diner B, Rochaix JD: Herbicide resistance in Chlamydomonas reinhardtii results from a mutation in the chloroplast gene for the 32-kilodalton protein of photosystem II. Proceedings of the National Academy of Sciences of the United States of America 1984, 81:3617-3621.

50. Hess FD, Bayer DE: Binding of the herbicide trifluralin to Chlamydomonas flagellar tubulin. Journal of Cell Science 1977, 24:351-360.

51. James SW, Ranum LPW, Silflow CD, Lefebvre PA: Mutants resistant to antimicrotubule herbicides map to a locus on the uni linkage group in Chlamydomonas reinhardtii. Genetics 1988, 118:141-147.

52. Strachan SD, Hess FD: The biochemical mechanism of action of the dinitroaniline herbicide oryzalin. Pesticide Biochemistry and Physiology 1983, 20:141-150.

53. Stavis RL, Hirschberg R: Phototaxis in Chlamydomonas reinhardtii. The Journal of Cell Biology 1973, 59:367-377.

54. Wakabayashi K-i, Misawa Y, Mochiji S, Kamiya R: Reduction-oxidation poise regulates the sign of phototaxis in Chlamydomonas reinhardtii. Proceedings of the National Academy of Sciences of the United States of America 2011, 108:11280-11284.

55. Cain JR, Cain RK: The Effects Of Selected Herbicides On Zygospore Germination And Growth Of Chlamydomonas Moewusii (Chlorophyceae, Volvocales). Journal of Phycology 1983, 19:301-305.

56. Heifetz P, Lers A, Turpin D, Gillham N, Boynton J, Osmond C: dr and spr/sr mutations of Chlamydomonas reinhardtii affecting D1 protein function and synthesis define two independent steps leading to chronic photoinhibition and confer differential fitness. Plant, Cell \& Environment 1997, 20:1145-1157.

57. Tóth R, van der Hoorn RAL: Emerging principles in plant chemical genetics. Trends in Plant Science 2010, 15:81-88.

58. Hall JL, Ramanis Z, Luck DJL: Basal body/centriolar DNA: molecular genetic studies in Chlamydomonas. Cell 1989, 59:121-132.

59. Tice CM: Selecting the right compounds for screening: does Lipinski\&apos;s Rule of 5 for pharmaceuticals apply to agrochemicals? Pest Management Science 2001, 57:3-16.

60. Tice CM: Selecting the right compounds for screening: use of surfacearea parameters. Pest Management Science 2002, 58:219-233.

61. Debenest T, Gagné F, Petit A-N, Kohli M, Eullafroy P, Blaise C: Monitoring of a flame retardant (tetrabromobisphenol A) toxicity on different microalgae assessed by flow cytometry. Journal of Environmental Monitoring 2010, 12:1918-1923.

62. Lamb J, Crawford ED, Peck D, Modell JW, Blat IC, Wrobel MJ, Lerner J, Brunet J-P, Subramanian A, Ross KN, Reich M, Hieronymus $\mathrm{H}$, Wei $\mathrm{G}$, Armstrong SA, Haggarty SJ, Clemons PA, Wei R, Carr SA, Lander ES, Golub TR: The Connectivity Map: using gene-expression signatures to connect small molecules, genes, and disease. Science 2006, 313:1929-1935.

63. Perlman ZE, Slack MD, Feng Y, Mitchison TJ, Wu LF, Altschuler SJ: Multidimensional drug profiling by automated microscopy. Science 2004, 306:1194-1198

64. Hoon S, Smith AM, Wallace IM, Suresh S, Miranda M, Fung E, Proctor M, Shokat KM, Zhang C, Davis RW, Giaever G, St Onge RP, StOnge RP, Nislow C: An integrated platform of genomic assays reveals smallmolecule bioactivities. Nature Chemical Biology 2008, 4:498-506.

65. PubChem. [http://pubchem.ncbi.nlm.nih.gov].

66. Chembank. [http://chembank.broadinstitute.org].

67. PharmGKB. [http://www.pharmgkb.org].

68. Wang Y, Duanmu D, Spalding MH: Carbon dioxide concentrating mechanism in Chlamydomonas reinhardtii: inorganic carbon transport and $\mathrm{CO} 2$ recapture. Photosynthesis Research 2011, 109:115-122.

69. Metzker ML: Sequencing technologies - the next generation. Nature Reviews Genetics 2010, 11:31-46.

70. Giaever G, Chu AM, Ni L, Connelly C, Riles L, Veronneau S, Dow S, LucauDanila A, Anderson K, Andre B, Arkin AP, Astromoff A, El-Bakkoury M, Bangham R, Benito R, Brachat S, Campanaro S, Curtiss M, Davis K, Deutschbauer A, Entian KD, Flaherty P, Foury F, Garfinkel DJ, Gerstein M, Gotte D, Guldener U, Hegemann JH, Hempel S, Herman Z, et al: Functional profiling of the Saccharomyces cerevisiae genome. Nature 2002, 418:387-391.
71. Oh J, Fung E, Schlecht U, Davis RW, Giaever G, St Onge RP, Deutschbauer A, Nislow C: Gene annotation and drug target discovery in Candida albicans with a tagged transposon mutant collection. PLoS Pathogens 2010, 6:e1001140.

72. Harris E: Chlamydomonas Handbook Academic Press, Inc., New York; 1989.

doi:10.1186/gb-2012-13-11-r105

Cite this article as: Alfred et al:: A phenotypic screening platform to identify small molecule modulators of Chlamydomonas reinhardtii growth, motility and photosynthesis. Genome Biology 2012 13:R105.

\section{Submit your next manuscript to BioMed Central and take full advantage of:}

- Convenient online submission

- Thorough peer review

- No space constraints or color figure charges

- Immediate publication on acceptance

- Inclusion in PubMed, CAS, Scopus and Google Scholar

- Research which is freely available for redistribution

Submit your manuscript at www.biomedcentral.com/submit
Ciomed Central 